\title{
»Boldly asserting our support of our friends and opposing with equal boldness our enemies « Die Ordnungsvorstellungen des amerikanischen Neokonservatismus für den Nahen und Mittleren Osten ${ }^{1}$
}

In Europa ist in der öffentlichen Wahrnehmung der so genannten »Neokonservativen « und ihres Einflusses auf die Außen- und Sicherheitspolitik der Bush-Administration nicht nur eine unscharfe Begriffsverwendung anzutreffen, mehr noch: Der Terminus hat weitgehend seine analytische Kraft verloren. Er hat stattdessen zum einen Anlass zu diversen Verschwörungstheorien geboten, in deren Kern eine neokonservative »Übernahme der amerikanischen Außenpolitik steht ${ }^{2}$ und ist zum anderen zu einem unreflektierten Kampfbegriff der politischen Debatte geworden wie in anderen Kontexten der Begriff des »Neoliberalismus«. Dies lässt sich nicht zuletzt daran ablesen, dass in den politischen Feuilletons und Kommentaren häufig Amtsträger zur Gruppe der Neokonservativen gezählt werden, die weltanschaulich mit diesen jedoch nichts gemein haben und stattdessen zur Gruppe der traditionellen Konservativen in den USA zu rechnen sind, z. B. Vize-Präsident Dick Cheney, Verteidigungsminister Donald Rumsfeld oder Außenministerin Condolezza Rice. ${ }^{3}$ Angesichts dieser Verwirrung ist es notwendig, erst einmal zu untersuchen, was sich substanziell hinter dieser politisierten Chiffre des Begriffs verbirgt. Zu fragen ist: Wer sind die so genannten Neokonservativen, auf welchem gesellschaftlichen Resonanzboden sind sie entstanden, was sind ihre außenpolitischen Ordnungsvorstellungen, und was ist das inhaltliche Band bezüglich der amerikanischen Außenpolitik, das ihre Zusammenfassung unter einen Begriff rechtfertigt? In einem zweiten Schritt sollen die neokonservativen Positionen zur amerikanischen Weltpolitik analysiert und am Beispiel des arabisch-israelischen Konfliktes und des Arabisch-Persischen Golf exemplifiziert werden. Zu fragen ist hier vor allem: Decken sie sich mit

1 Der Autor dankt Johanna Nemson für ihre wertvolle Unterstützung bei den Recherchen für diesen Aufsatz sowie den Teilnehmern der Jahrestagung 2003 der Sektion Politikwissenschaft der Deutschen Gesellschaft für Amerikastudien, insbesondere PD Dr. Peter Rudolf, für zahlreiche Anregungen und konstruktive Kritik.

2 Für einen guten Überblick über diese und entsprechende Repliken vgl. Joshua Muravchik, »The Neoconservative Cabal« in: Commentary, 2/2003, S. 26-33, Robert J. Lieber, »The Neoconservative-Conspiracy Theory: Pure Myth« in: The Chronicle Review, 34/ 2003, in: <http://chronicle.com/free/v49/i34/34b01401.htm> und Max Boot, »Think Again: Neocons « in: Foreign Policy, 1/2004, S. 20-28.

3 Diese fehlerhafte Analyse ist jedoch nicht auf Europa beschränkt. Vgl. z. B. Zbigniew Brzezinski, »Europäer in den Irak! Die Zukunft der transatlantischen Beziehungen« in: Internationale Politik, 8/2003, S. 57-66 (S. 59). 
der Politik der Bush-Administration, wie sie nach außen hin erkennbar wird, d. h. besitzen die Neokonservativen eine dominierende oder sogar ausschließliche Gestaltungsmacht in diesem Politikfeld? Schließlich gilt es, das Verhältnis dieser Ordnungsvorstellungen zu denen relevanter gesellschaftlicher Gruppen und Akteure in den USA zu diskutieren und zu fragen, wie diese sich gegenseitig beeinflussen. Abschließend soll vorsichtig darüber spekuliert werden, welche Rolle die Neokonservativen in der zweiten Amtszeit von Präsident Bush spielen könnten.

\section{Zum außenpolitischen Profil der amerikanischen Neokonservativen}

Ursprünglich waren die Neokonservativen Teil einer breiten anti-kommunistischen Koalition, die den amerikanischen Liberalismus von den späten 1940er bis zu den späten 1960er Jahren dominierte. Sie lehnten dann aber die sozial- und außenpolitische Linkswende des Liberalismus und der Demokratischen Partei in den 1970er Jahren ab; einzelne Vertreter des amerikanischen Neokonservatismus, z. B. Richard Perle, sind jedoch bis heute Mitglieder der Demokratischen Partei. Vor diesem Hintergrund ist auch Irving Kristols berühmtes Zitat zu verstehen, einem der einflussreichsten Gründungsfiguren der Neokonservativen, der einen Neokonservativen definierte "... as a liberal who has been mugged by reality."

Bei diesen Vertretern handelt es sich um eine Gruppe von Intellektuellen, die recht unterschiedliche Ordnungsvorstellungen vertreten: Während die einen in sozialen Fragen wohlfahrtsstaatlichen Modellen gegenüber offen sind und auch wirtschaftspolitische Interventionen des Staates befürworten, gibt es daneben andere, die Marktintervention streng ablehnen. Einige vertreten in der Außenpolitik einen aggressiven Interventionismus, während andere, vor allem in der Anfangszeit des Neokonservatismus, eine eher zurückhaltende Anwendung amerikanischer Macht befürwortet haben. Die Gruppe hat weder ein gemeinsames Programm, noch ist sie durch Manifeste gebunden, aus denen sich ein politisches Credo ableiten ließe. Und auch der Versuch einiger europäischer Beobachter, eine gemeinsame akademische Sozialisation zu konstruieren, geht fehl: Nicht alle Neokonservative haben bei denjenigen akademischen Lehrern studiert, die häufig als intellektuelle Väter dieser Strömung benannt werden: Allan Bloom, Albert Wohlstetter und Leo Strauss. ${ }^{4}$

4 So z. B. Heinrich August Winkler, der Leo Strauss als geistigen Urheber der Neokonservativen identifiziert: "Wenn die Macht Recht spricht « in: Die Zeit, 18. 6. 2003. Auch James Atlas ist in der New York Times dieser Lesart gefolgt und hat die "Neo-Cons « als "Leo-Cons « apostrophiert: »A Classicists Legacy: New Empire Builders « in: The New York Times, 4. 5. 2003. Vgl. dazu weitgehend zustimmend Christian Geyer, »Der doppelte Bush « in: Frankfurter Allgemeine Zeitung, 18. 6. 2003. Dagegen skeptisch (und sehr viel differenzierter) Henning Ritter, »Das Orakel von Chicago « in: Frankfurter Allgemeine Sonntagszeitung, 29. 6. 2003 sowie Richard Herzinger, "Spinnen im Netz der amerikanischen Weltherrschaft « in: Die Zeit, 3. 7. 2003. Aus neokonservativer Perspektive haben zuletzt Steven Lenzner und William Kristol die unmittelbare Relevanz von Strauss für die außenpolitischen Ordnungsvorstellungen der Neokonservativen zurückgewiesen: »What Was Leo Strauss Up To?« in: The Public Interest, 153/2003, S. 19-39. 
Zwei Dinge eint diese Gruppe jedoch: zum einen ihr gemeinsames Selbstverständnis als Intellektuelle, die ihre Ordnungsvorstellungen in der Politik umzusetzen suchen, zum Zweiten ihr tief verwurzelter Glauben an die Überlegenheit der amerikanischen Werte und die Notwendigkeit, diesen aus sicherheitspolitischen Erwägungen heraus global Geltung zu verschaffen. Mit dieser Orientierung unterscheiden sie sich von traditionellen Konservativen, die nach dem Debakel des Vietnam-Krieges zumeist für den Rückzug der USA aus der Weltpolitik eintraten. ${ }^{5}$ Dass diese Wertorientierung nicht bloße Rhetorik ist, lässt sich z. B. an der Rolle von Paul Wolfowitz, des ehemaligen stellvertretenden Verteidigungsministers, bei den Regimewechseln in Indonesien und auf den Philippinen ablesen: In seinen Funktionen als Assistant Secretary of State for East Asian and Pacific Affairs und als US-Botschafter in Indonesien während der 1980er Jahre unterstützte er die Opposition maßgeblich dadurch, indem er den Machthabern signalisierte, dass sie nicht länger mit der Duldung ihrer Menschenrechtsverletzungen durch die USA rechnen könnten, und oppositionelle Gruppen ermutigte. ${ }^{6}$

Diese Fokussierung auf die amerikanischen Werte resultiert vor allem aus der wichtigsten neokonservativen außenpolitischen Bezugsgröße: der totalitären Erfahrung Europas und der Welt im 20. Jahrhundert. Sie ist nach wie vor eine treibende und lebendige politische Kraft, die der entscheidende Grund dafür ist, dass die Neokonservativen das heutige internationale System bevorzugt in Kategorien von Gut und Böse interpretieren und aktuelle Akteure in historischen Parallelen betrachten: So ist es nahezu zwangsläufig, dass für diese ideologische Strömung Saddam Hussein, Kim Jong Il und Fidel Castro nicht nur Diktatoren sind, ohne die die Welt ein besserer Ort wäre, sondern sie stehen für die Neokonservativen in einer unmittelbaren Tradition zu Hitler und Stalin. Diese historisierende Weltsicht erstreckt sich auch auf die Debatte, welche Strategien gegenüber solchen Akteuren einzuschlagen seien. So fasste z. B. ein Artikel von William Kristol im Weekly Standard die Kritiker der Irak-Politik Präsident George W. Bushs unter dem Begriff der »Axis of Appeasement " zusammen. ${ }^{7}$

Im Gegensatz zu den klassischen Realisten, die ausschließlich auf Grund amerikanischer Interessen argumentieren, sind die Neokonservativen mit ihrem Bezug auf amerikanische Werte im Kern idealistisch. Häufig werden sie daher in einer Tra-

5 Die Unterscheidung zwischen klassischen oder »realistischen « Konservativen und den Neokonservativen in der amerikanischen Außenpolitik soll an dieser Stelle nicht weiter vertieft werden. Vgl. dazu Gerard Baker/ Stephen Fidler, »America's Democratic Imperialists: How the Neo-Conservatives Rose from Humility to Empire in Two Years « in: Financial Times, 6. 3. 2003.

6 Zu einem Profil Wolfowitz’ vgl. Bernd Kubbig, Wolfowitz' Welt verstehen: Entwicklung und Profil eines »demokratischen Realisten", Frankfurt/M 2004.

7 William Kristol, »The Axis of Appeasement « in: The Weekly Standard, 26. 8. 2002, S. 78. Zur Rolle des Weekly Standard als neokonservativem Ideengeber der Bush-Administration vgl. Susanne Klingenstein, »Alle Wege führen zu Bush. Höre und herrsche: Wer den Präsidenten auf Ideen bringt« in: Frankfurter Allgemeine Zeitung, 1. 11. 2003. 
dition zu US-Präsident Woodrow Wilson gesehen und begreifen sich z. T. selbst so. ${ }^{8}$ Dies ist bei genauerer Betrachtung jedoch kaum zu halten, denn Wilson war weitgehend - in den Kategorien der Theorien der internationalen Beziehungen - Realist und bis in die erste Phase seiner Amtszeit hinein sogar Imperialist. Er wandelte seine Positionen später aber und plädierte dann für ein vermittelndes System von internationalem Recht und Organisationen, das die Macht großer Staaten bändigen, die demokratische Selbstbestimmung sowie die Souveränität und Unabhängigkeit kleinerer und schwächerer Nationen schützen solle - etwas, was die heutigen Neokonservativen für die USA gerade eben nicht akzeptieren und stattdessen von einer ungleichen Legitimität von Staaten ausgehen. Zudem warnte Wilson immer vor den nationalistischen Gegenreaktionen, die der amerikanische Imperialismus hervorriefe. Und schließlich würden die Neokonservativen Wilsons »Making the World for Democracy« eher als »Making the World Safe by Democracy« paraphrasieren, also die instrumentelle Bedeutung von Demokratisierung betonen. ${ }^{9}$ Damit sind sie - zumindest implizit - Anhänger der Theorien des demokratischen Friedens, die die pazifizierende Wirkung von Demokratisierung auf das außenpolitische Verhalten von Staaten betonen. ${ }^{10}$ In diesem Sinne sind die neokonservativen Protagonisten - im Gegensatz zu den »klassischen« Konservativen - zudem auch immer Befürworter des nation-building gewesen. ${ }^{11}$

Ihren politischen Durchbruch erlebten die Neokonservativen seit 1980 mit der Umsetzung von Teilen ihrer Ordnungsvorstellungen unter Präsident Reagan, dessen Republikanischer Partei sie sich zugewandt hatten. Doch mit dem Ende von dessen Regierungszeit sowie der Aufhebung des Ost-West-Konfliktes verloren sie an Bedeutung, und die Bush I-Administration verfolgte eine Außenpolitik, die sie als zu weich gegenüber der Sowjetunion, als zu inkonsequent gegenüber Saddam Hussein im Zweiten Golfkrieg 1991 und lange Zeit als zu zurückhaltend im Bosnien-Konflikt ablehnten. Für die Neokonservativen war wieder einmal eine amerika-

8 Vgl. zu dieser Debatte u. a. Colin Dueck, »Hegemony on the Cheap. Liberal Internationalism from Wilson to Bush « in: World Policy Journal, 4/2003, S. 1-11; Klaus-Dieter Frankenberger, »Idealismus reicht nicht aus. Woodrow Wilson als geistiger Vater des Neokonservativismus in Amerika? in: Frankfurter Allgemeine Zeitung, 25. 11. 2003 und Tim B.Müller, »Falsche Erben. Amerikas Neokonservative behaupten, in Woodrow Wilsons Fußstapfen zu treten, doch davon kann keine Rede sein« in: Süddeutsche Zeitung, 4. 6. 2003.

9 Diesen Hinweis verdanke ich meinem Jenaer Kollegen Manuel Fröhlich. Vgl. dazu auch Boot, "Think Again: Neocons « aaO. (FN 2), S. 24: "Neocons believe the United States should use force when necessary to champion its ideals as well as its interests, not only out of sheer humanitarianism but also because the spread of liberal democracy improves U.S. security, while crimes against humanity inevitably make the world a more dangerous place."

10 Vgl. John B. Judis, »What Woodrow Wilson Can Teach Today's Imperialists « in: The New Republic, 9. 6. 2003 sowie Jedediah Purdy, »Liberal Empire. Assessing the Arguments in: Ethics and International Affairs, 2/2003, in: <www.cceia.org/viewMedia.php/prmTemplateID/8/prmID/1026>.

11 Vgl. Tim B.Müller, »Ihr Feind heißt Rumsfeld. Die amerikanischen Neokonservativen sind gespalten« in: Süddeutsche Zeitung, 20. 3. 2004. 
nische Administration ihrer weltpolitischen Verantwortung, ja Verpflichtung nicht gerecht geworden ${ }^{12}$, wieder einmal waren die amerikanischen Werte verraten und die Chance vergeben worden, dass die USA als moralische Führungsmacht die freie Welt in den Kampf gegen das Böse führten. ${ }^{13}$ In dieser Kritik klingt die verbindende außenpolitische Ordnungsvorstellung der Neokonservativen nach dem Ende des Ost-West-Konfliktes an: Das unipolare Moment, d. h. die unangefochtene Machtstellung der Vereinigten Staaten im internationalen System nach 1989/90, an die kein anderer Akteur des internationalen Systems in absehbarer Zukunft heranreiche, müsse nutzbar gemacht werden, um die amerikanische Macht in dieser veränderten Struktur der Weltpolitik ungebändigt zur Geltung zu bringen. ${ }^{14}$

Bei der Entfremdung der Neokonservativen von der Republikanischen Partei hatte die amerikanische Nahostpolitik von George H. W. Bush eine zentrale Rolle gespielt. Denn im Verlauf des im Oktober 1991 begonnenen arabisch-israelischen Friedensprozesses hatte die Bush-Administration begonnen, Druck auf die israelische Regierung auszuüben und in diesem Zusammenhang die Gewährung einer Kreditgarantie über zehn Milliarden Dollar an Israel, die für Siedlungsprogramme für aus Russland eingewanderte Juden vorgesehen waren, durch den US-Kongress verhindert. Auch die zwar im kleinen Kreis getätigte, aber an die Presse lancierte Äußerung von Außenminister James Baker "Fuck the Jews. They didn't vote for us" verstärkte den Eindruck der Neokonservativen, dass ein nahostpolitischer Kurswechsel zugunsten Israels nur mit einem neuen Präsidenten möglich sei.

Die Distanziertheit der Neokonservativen zur Bush I-Administration ließ sich erneut an der amerikanischen Irak-Debatte der Jahre 2002/2003 ablesen. Skeptiker aus dieser Regierung wie Brent Scowcroft oder James Baker, die den Kriegskurs der USA kritisch kommentierten, zogen in besonderer Weise den Spott neokonservativer Kommentatoren auf sich. Europäische Kritik an den Neokonservativen, die auf die bürokratischen Kontinuitäten zwischen Bush Sr. und Bush Jr. zielt, geht also in der Sache völlig fehl. Die Ablehnung von Bush Sr. ist auch ein Grund, warum viele Neokonservative während des Wahlkampfes und den ersten Monaten seiner Amts-

12 David Calleo hält den Gedanken der »responsibility« für den Kern neokonservativer, außenpolitischer Ordnungsvorstellungen: "Faith in their own strength puts them in the uncomfortable moral position of an omnipotent god, to wit: If there is evil in the universe, it is America's fault for not removing it. "David P. Calleo, »Power, Wealth and Wisdom. The United States and Europe after Iraq in: The National Interest, 72/2003, S. 5-15 (S. 7). Max Boot spricht sogar von »destiny«. Vgl. ders., »America’s Destiny Is to Police the World « in: Financial Times, 18. 2. 2003.

13 Ohne dass die Neokonservativen selbst religiös inspiriert wären, eint diese dualistische Weltsicht sie mit Vertretern religiöser Gruppierungen in den USA, die ähnliche Ordnungsvorstellungen propagieren. Auch die religiöse Vorstellungswelt von George W. Bush selbst lässt sich mühelos mit neokonservativen Idealen in Deckung bringen. Vgl. Andrew J. Bacevich, / Elizabeth H. Prodromou, »God is Not Neutral: Religion and U.S. Foreign Policy after 9/11 in: Orbis, 1/2004, S. 43-54 (S. 50f.).

14 Vgl. dazu Charles Krauthammer, »The Unipolar Moment « in: Foreign Affairs, 1/1991, S. 23-33 sowie ders., »The Unipolar Moment Revisited « in: The National Interest, 70/ 2002-03, S. 5-17. 
zeit Bill Clinton trotz der Tatsache Interesse und Sympathie entgegenbrachten, dass er mit Warren Christopher und Anthony Lake zwei Vertreter der von den Neokonservativen verachteten Carter-Administration für die Posten des Außenministers und des Nationalen Sicherheitsberaters in seiner Regierung gewonnen hatte. ${ }^{15} \mathrm{Zu}$ dem schien er auf den ersten Blick mit vielen ihrer Ordnungsvorstellungen überein zu stimmen: Der Gouverneur von Arkansas hatte während des Wahlkampfes 1992 dem Amtsinhaber außenpolitische Schwäche vorgeworfen, so z. B. in der Frage von Waffenlieferungen an Bosnien, der Politik gegenüber dem Irak vor und nach dem Ende der Besetzung Kuwaits sowie bezüglich der amerikanischen Reaktion auf die blutige Unterdrückung der chinesischen Studentenunruhen im Sommer 1989. Als eigene Schwerpunkte hatte er die Verpflichtung zur globalen Verbreitung der Demokratie (»Enlargement«), die Beibehaltung einer starken nationalen Verteidigung und die Bereitschaft zur Anwendung von militärischer Gewalt, sofern nötig, betont. Zudem hatte er seinen Willen bekundet, die amerikanischen Sicherheitsinteressen gegen neue Herausforderungen zu verteidigen und dabei die Proliferation von Massenvernichtungswaffen genannt. Mit diesem Programm setzte er erste außenpolitische Schwerpunkte, die viele neokonservative Vertreter nicht nur teilen konnten, sondern die Kernpunkte ihrer Kritik an Präsident Bush gewesen waren. Doch bereits nach wenigen Monaten von Clintons Amtszeit endete die kurze Sympathie der Neokonservativen für die neue Administration, zum einen, da zahlreiche Neokonservative, die auf eine Tätigkeit in der Exekutive gehofft hatten, enttäuscht worden waren, und zum Zweiten, da die Clinton-Administration in ihren ersten außenpolitischen Bewährungsproben (Bosnien, Haiti, Somalia) ihren Ankündigungen nicht gerecht werden konnte. ${ }^{16}$

Die außenpolitische Äußerung der Neokonservativen mit der größten Resonanz war das »Defense Policy Guidance Paper" von Paul Wolfowitz aus dem Jahr 1992, das eine unangefochtene Stellung der Vereinigten Staaten im internationalen System postulierte, die globale Durchsetzung amerikanischer Interessen empfahl sowie den Aufstieg potenzieller machtpolitischer Rivalen als die zentrale zu verhindernde Entwicklung des 21. Jahrhunderts beschrieb. Für die Neokonservativen ist demzufolge die anzustrebende Weltordnung diejenige, in der die USA ihre uneingeschränkte Macht durchsetzen, die unipolare Weltordnung zum Ausgangspunkt und zugleich Ziel aller strategischen Überlegungen machen und dabei auf die Einwände und Bedenken anderer Akteure wenig oder keine Rücksicht nehmen; oder wie William Kristol es formulierte: "... a morally grounded foreign policy that seeks aggressively and unapologetically to advance American principles around the world. «17 Die Kodifikation ihrer außenpolitischen Ordnungsvorstellungen erreichten die Neokon-

15 Vgl. beispielhaft den Aufsatz von Paul Wolfowitz, »Clinton's First Year« in: Foreign Affairs, 1/1994, S. 28-43, der in nahezu schwärmerischem Ton Clinton als einen neuen Truman zeichnet, der die USA durch eine stürmische Phase der weltpolitischen Neuorientierung führen könne.

16 Vgl. John Ehrman, The Rise of Neoconservatism. Intellectuals and Foreign Affairs, 1945-1994, New Haven/London 1995, besonders S. $193 \mathrm{ff}$.

17 Kristol, »The Axis of Appeasement « aaO. (FN 7). 
servativen mit der so genannten Bush-Doktrin, d. h. der Nationalen Sicherheitsstrategie vom September 2002. Sie folgt einer neokonservativen Weltanschauung des notwendigen, ja von den USA geforderten Aktivismus insofern, als sie betont, dass in dem seit dem 11.9.2001 veränderten Bezugsrahmen der amerikanischen Politik die Instrumente des Kalten Krieges, nämlich Eindämmung und Abschreckung, nicht mehr geeignet und nunmehr präemptive Schläge geboten seien.

Die angesprochene konzeptionelle Bezugnahme auf die Außenpolitik der Reagan-Administration unter gleichzeitiger Ablehnung der Außenpolitik Präsident Clintons sowie der traditionellen Konservativen gleichermaßen spiegelt sich in einem der wichtigsten neokonservativen Dokumente wider, der Prinzipienerklärung des Project for the New American Century vom 03. Juni 1997.18 In dieser kritisieren zahlreiche Entscheidungsträger der Reagan-Administration, die unter George W. Bush wieder wichtige Posten in der Administration bekleiden sollten, u.a. Elliott Abrams, Richard Cheney, Zalmay Khalilzad, Peter W. Rodman und Donald Rumsfeld, die Politik Clintons und entwerfen Paradigmen für eine neue amerikanische Außenpolitik: Auf Grund ihrer einzigartigen Machtsstellung im internationalen System und der neuen Herausforderungen seien die Vereinigten Staaten in besonderer Weise zu einer globalen Führungsrolle aufgefordert, die die Umsetzung amerikanischer Prinzipien und Werte zum Ziel haben müsse. Diese strategische Grundannahme solle sich in vier Konsequenzen niederschlagen, nämlich einer Erhöhung der Verteidigungsausgaben und der Modernisierung der amerikanischen Streitkräfte, im Ausbau der Beziehungen zu denjenigen amerikanischen Verbündeten, die die Prinzipien der Demokratie teilen und in einer Bekämpfung derjenigen Regime, die diese Prinzipien herausfordern; im globalen Kampf für politische und ökonomische Freiheit sowie in der Akzeptanz einer amerikanischen Führungsrolle zur Herstellung einer internationalen Ordnung, die die Sicherheit der USA, ihre ökonomische Prosperität sowie die Durchsetzung amerikanischer Prinzipien begünstige.

$\mathrm{Zu}$ Beginn der Amtszeit George W. Bushs besaßen die Neokonservativen aber keine erkennbare ideologische Schlüsselrolle innerhalb der Administration, die ihre Ordnungsvorstellungen besonders exponiert hätte hervortreten lassen. Erst die Anschläge des 11. September ließen die Notwendigkeit einer neuen, umfassenden Strategie hervortreten, die die Stellung der USA im internationalen System neu definierte und eine angemessene Antwort auf die neue Herausforderung durch den transnationalen Terrorismus bot. ${ }^{19}$ Die Neokonservativen hatten in den Jahren zu-

18 Vgl.: <http://www.newamericancentury.org/statementofprinciples.htm>. Nicht alle Unterzeichner dieser Erklärung sind den Neokonservativen zuzurechnen. Sie enthält jedoch einen Kanon außenpolitischer Prinzipien, dem alle Neokonservativen zustimmen können. Vgl. zur Bedeutung der Außenpolitik der Reagan-Administration für die Neokonservativen auch William Kristol / Robert Kagan, »Toward a Neo-Reaganite Foreign Policy« in: Foreign Affairs, 4/1996, S. 18-32.

19 Vgl. zur Wirkung neokonservativer Ordnungsvorstellungen auf die Außenpolitik der Bush II-Administration Alexandra Homolar-Riechmann, »Pax Americana und gewaltsame Demokratisierung. Zu den politischen Vorstellungen neokonservativer Think Tanks « in: Aus Politik und Zeitgeschichte, B 46/2003, S. 33-40; Sam Tanenhaus, »Bush’s Brain Trust « in: Vanity Fair, July 2003, S. 114-118 u. 164-169; Walter Laqueur, »Der Mythos der ,NeoCons" "in: Europäische Rundschau, 2/2003, S. 3-9 und Uwe Schmitt, »Mythenwesen oder Revolutionäre? Amerikas Neo-Konservative im Umfeld von Bush« in: Die Welt, 12. 5. 2003. 
vor immer wieder auf die Gefahr terroristischer Anschläge sowie der Proliferation von Massenvernichtungswaffen verwiesen und waren nicht zuletzt deshalb für eine Erhöhung des Verteidigungsetats eingetreten. Mit den Anschlägen des 11. Septembers durften sie sich bestätigt sehen. Sie waren nun mit ihrem »robusten Idealismus«, der Passivität für gefährlicher als Aktivismus hält, also mit einer zuvor bereits existierenden Strategie zur richtigen Zeit am richtigen Ort. Anders formuliert: Ohne den 11. September wäre es fraglich, ob die politische Öffentlichkeit in Europa überhaupt einen »neoconservative turn " der amerikanischen Außenpolitik diskutierte und dessen Vertreter gezielt wahrnähme.

\section{Die Neokonservativen und der arabisch-israelische Konflikt}

Zentral für die außenpolitischen Ordnungsvorstellungen der Neokonservativen ist ihr vorbehaltloses Engagement für Israel als der einzigen Demokratie westlichen Maßstabs im Nahen und Mittleren Osten, ihre politische Orientierung an den Leitideen der Likud-Partei sowie ihr Festhalten an einem amerikanischen Engagement im Nahen Osten. Dabei stehen sie dem Madrider Friedensprozess, den seit 1993 erreichten Oslo-Vereinbarungen, die einen politischen und territorialen Ausgleich zwischen Israel und den Palästinensern zum Ziel haben, und der Politik der Bush Isowie der Clinton-Administration skeptisch bis ablehnend gegenüber. Statt einer engen Fokussierung der amerikanischen Politik auf eine Regelung des arabisch-israelischen Konfliktes postulieren neokonservative Vertreter die Sicherheit und die Machtposition der USA und ihrer regionalen Verbündeten als Bezugspunkt der amerikanischen Nahostpolitik. ${ }^{20}$ Denn erst die dominierende Stellung der USA im Nahen Osten nach dem Ende des Ost-West-Konfliktes und dem Zweiten Golfkrieg 1991 habe eine regionale Kräfteverschiebung zur Folge gehabt, die den Madrider Friedensprozess ermöglicht habe. Dies bedeute in der Konsequenz auch, Abschied von dem Anspruch zu nehmen, als scheinbar unparteiischer Vermittler zwischen Israel und den Palästinensern zu vermitteln und sich stattdessen klar zu den engen amerikanisch-israelischen Beziehungen zu bekennen. Dies hätten sowohl die Bush I- als auch die Clinton-Administration unterlassen und damit die Regierungen in Jerusalem während der 1990er Jahre zu Konzessionen gegenüber der palästinensischen Autonomiebehörde gedrängt, die auf Grund der Politik der Palästinenser nicht gerechtfertigt gewesen wären und damit Israel letztlich geschadet hätten. So hätten Bush und Clinton bzw. der amerikanische Sondervermittler Ross die palästinensischen Verstöße gegen die Oslo-Verpflichtungen, die Menschenrechtsverletzungen in den palästinensischen Autonomiegebieten sowie den autoritären Charakter des politischen Systems mit Präsident Arafat an der Spitze aus ideologischen

20 Vgl. dazu und dem Folgenden den Aufsatz von Elliott Abrams, des seit Dezember 2002 höchsten Nahostberaters im National Security Council: »Israel and the ,Peace Process' " in: Robert Kagan / William Kristol (Hg.), Present Dangers. Crisis and Opportunity in American Foreign and Defense Policy, San Francisco 2000, S. 221-240. Für ein Profil dieses Neokonservativen vgl. Michael Dobbs, »Reagan-Era Infighter Returns to Help Set U.S. Foreign Policy« in: Wall Street Journal Europe, 28. 5. 2003. 
Gründen nicht ausreichend kritisiert. Bezüglich der israelisch-syrischen Konfliktkonstellation fordern die Neokonservativen den Rückzug Syriens aus dem Libanon und eine Stärkung der seit 1996 bestehenden israelisch-türkischen Militärkooperation als Schutz gegenüber Damaskus. Neben diplomatischen Initiativen sehen sie ebenso in der Androhung militärischer Gewalt ein wirksames Instrument amerikanischer Nahostpolitik. ${ }^{21}$

Sie sind mit dieser Politik bemerkenswerterweise in weiten Teilen »israelischer« als dies amerikanische Juden traditionell sind, die überwiegend den Demokraten anhängen und von denen auch bei den Präsidentschaftswahlen 2000 lediglich 19\% George W. Bush gewählt haben. Festzustellen ist mittlerweile jedoch eine Erosion dieser klaren politischen Identifikation: Denn weite Teile der jüdischen Bevölkerungsgruppe nähern sich der Republikanischen Partei und den nahostpolitischen Positionen der Neokonservativen an, denen sie eigentlich fern stehen. ${ }^{22}$ Vor allem die seit September 2000 anhaltende Gewalt im Verlauf der zweiten palästinensischen Intifada und die antiisraelische Zielrichtung des islamistischen Terrorismus haben unter amerikanischen Juden eine Verbundenheit mit Israel ausgelöst, die existierende parteipolitische Differenzen überdeckt. Zwar haben sich zwei Drittel der amerikanischen Juden prinzipiell für einen palästinensischen Staat ausgesprochen - eine Position, die sie nicht gerade in die Arme Ariel Scharons treibt. Dennoch vertreten sie mittlerweile kompromisslose Positionen in der Frage, wie mit der palästinensischen Führung umzugehen sei und treffen sich dabei mit neokonservativen Einstellungen. Damit vollzieht sich ideologisch in den USA nahezu spiegelbildlich eine Entwicklung, die auch in Israel festzustellen ist. ${ }^{23}$

Allerdings haben sich diese veränderten Präferenzen der amerikanischen Juden bei den Wahlen 2004 kaum in einem veränderten Wahlverhalten niedergeschlagen: Bei den jüngsten Wahlen lag der Anteil der republikanischen Wähler in dieser Gruppe mit 25\% nur unerheblich höher als im Jahr 2000. Der Bush-Administration ist es also nur begrenzt gelungen, in dieser Bevölkerungsgruppe ihren Stimmanteil zu erhöhen, wofür ihre Israel- und Irak-Politik doch eigentlich wirksame Vehikel gewesen wären. Ein möglicher Grund dafür ist, dass sie weiterhin die arabischstämmigen Amerikaner als Wählerpotential anzusprechen sucht, von denen 45\% Bush im Jahr 2000 ihre Stimme gaben. Allerdings haben bei den Präsidentschafts-

21 Abrams, ebd., S. 239: "The next decade will present enormous opportunities to advance American interests in the Middle East, but not for the most part through painstaking negotiations of documents. Here, as elsewhere, the principal means of asserting our national interests will be boldly asserting our support of our friends and opposing with equal boldness our enemies. Here, as elsewhere, much depends on judgements others make about our ability to see our interests clearly and our resolve to protect them. Here, as elsewhere, our military strength and willingness to use it will remain a key factor in our ability to promote peace.«

22 Vgl. Eva Schweitzer, »Die Angst wächst in Manhattan. Warum viele New Yorker Juden aus ihrem traditionell liberalen in ein neokonservatives Milieu gewechselt sind « in: Die Zeit, 8. 1. 2004.

23 Vgl. Ofira Seliktar, Divided We Stand. American Jews, Israel and the Peace Process, Westport/London 2002. 
wahlen 2004 lediglich 29\% dieser Gruppe für Bush gestimmt. Dafür werden einerseits der in der ersten Amtszeit ausgebliebene Druck auf Israel und andererseits die für die Arab-Americans negativen Folgen des Patriot Act verantwortlich sein.

Mit ihrer Unterstützung der israelischen Rechten finden Neokonservative zudem unter den evangelikalen Gruppen, d.h. christlichen Fundamentalisten des »Bible Belt« des amerikanischen Südens, die aus religiösen Gründen Israel engagiert unterstützen, große Resonanz: 78\% dieser Gruppe haben bei den jüngsten Präsidentschaftswahlen für George W. Bush gestimmt.

Umgekehrt dazu steht die Führung der palästinensischen Autonomiebehörde im besonderen Fokus neokonservativer Kritik. Diese sei kein zu akzeptierender Verhandlungspartner der USA, solange sie z. B. Waffen vom Iran importiere, einem Staat, den das State Department seit Jahren auf der Liste der Länder führt, die den internationalen Terrorismus unterstützen, und zugleich palästinensischen Terrorgruppen in ihrem Kampf gegen Israel freie Hand lasse. Dementsprechend treten neokonservative Kommentatoren entweder für eine völlige Streichung aller amerikanischen Finanzhilfen an die palästinensische Autonomiebehörde ein, oder aber sie verlangen eine strikte Konditionalisierung, d. h. die Kopplung der Hilfen an ein verifizierbares Ende palästinensischer Terrorangriffe. Doch selbst wenn die Palästinenser und andere arabische Akteure sich verhandlungs- und konzessionsbereit zeigen, weckt dies bei einigen Neokonservativen Skepsis: So argumentierte Douglas Feith, heute Under Secretary of Defense for Policy, im Jahr 1994, also zu einer Hochzeit des Friedensprozesses, dass in der Geschichte alle expansiven Diktatoren, u. a. Adolf Hitler, sich einmal auf Friedensverhandlungen eingelassen hätten, um damit aber nur ihre wahren Absichten zu verschleiern - ein neokonservatives Argument reinster Provenienz. ${ }^{24}$

Einige neokonservative Extrempositionen, wie z. B. die von David Wurmser, des Nahostexperten des American Enterprise Institute, lehnen den arabisch-israelischen Friedensprozess aus prinzipiellen Erwägungen völlig ab, da dieser weder Frieden für die beteiligten Akteure noch den USA irgendwelche Sympathien in der Region gebracht habe, im Gegenteil: Die Clinton-Administration habe sich von totalitären Systemen wie Syrien und der palästinensischen Autonomiebehörde vorführen lassen und damit alle amerikanischen Werte verleugnet. Das Ergebnis der jahrzehntelangen Bemühungen sei zudem eine Welle des Antiamerikanismus und Antiisraelismus in der arabischen Welt, die unvergleichbar sei. Solange die Zentren des arabischen Radikalismus nicht ausgeschaltet seien, und hier nennt Wurmser namentlich Damaskus, Bagdad, Tripolis, Teheran und Gaza, werde es keine regionale Ordnung amerikanischer Prägung geben..$^{25}$

24 Douglas Feith, »Land for No Peace« in: Commentary, 6/1994, S. 32-36.

25 David Wurmser, »Middle East War« in: Washington Times, 1. 11. 2000, in: <http:// www.aei.org/news/newsID.12107,filter./news_detail.asp >: »That would reestablish the recognition that fighting with either the United States or Israel is suicidal. Many in the Middle East will then understand the merits of being an American ally and of making peace with Israel." 


\section{Neokonservative Ordnungsvorstellungen für den Arabisch-Persischen Golf}

Die Anschläge des 11. September 2001 haben existierende Perzeptionen unter den Neokonservativen, dass nämlich der Irak einer der dominierenden außenpolitischen Bedrohungen der amerikanischen Interessen am Arabisch-Persischen Golf sei, lediglich bestätigt und noch verstärkt. Auch lässt sich berechtigt darüber spekulieren, ob ohne den 11. September die Bush-Administration die notwendige innenpolitische Unterstützung für den Irak-Krieg hätte generieren können. So hatte noch im Sommer 2001 Außenminister Powell im VN-Sicherheitsrat ein verbessertes Sanktionsregime, die so genannten smart sanctions durchgesetzt. Und die gegenwärtige Außenministerin Condoleeza Rice hatte während des Wahlkampfes 2000 angekündigt, dass die Politik der Abschreckung gegenüber Bagdad weiterhin die erfolgversprechendste Strategie gegen den Einsatz irakischer Massenvernichtungswaffen und die Grundlage der Politik der Bush-Administration sein werde. ${ }^{26}$ In der ersten Monaten ihrer Amtszeit gab es also noch keine Mehrheit innerhalb der Administration für einen Paradigmenwechsel in der Irak-Politik, im Gegenteil: Die seit zehn Jahren andauernde Politik des Containment wurde auch unter der dritten Administration fortgesetzt.

Unmittelbar nach den Anschlägen fand nun aber Paul Wolfowitz mit seinem Plädoyer, Saddam Husseins Regime zu stürzen, das Ohr des Präsidenten und konnte früheren Konzeptionen den Weg bahnen. Denn bereits 1998 hatten acht Neokonservative, die in der Bush II-Administration wichtige Posten einnehmen sollten, darunter Wolfowitz und Richard Perle, einen offenen Brief an Präsident Clinton verfasst, in dem sie die Erfolglosigkeit der Clintonschen Eindämmungsstrategie beklagten und einen Regimewechsel in Bagdad als einzige effiziente Form qualifizierten hatten, der irakischen Bedrohung mit Massenvernichtungswaffen Herr zu werden. Schon 1999 hatte Wolfowitz die Entsendung amerikanischer Bodentruppen in den Südirak und die Einrichtung einer Sicherheitszone gefordert, in der die irakische Opposition den Sturz Saddam Husseins vorbereiten könne. ${ }^{27}$ Und ein Jahr später hatte Perle seine Kritik an der Politik der Clinton-Administration dahingehend spezifiziert, dass er auf die zentrale Bedeutung der irakischen Exilopposition für einen Regimewechsel hingewiesen und zugleich die mangelnde Unterstützung durch die Exekutive trotz entsprechender Vorgaben des US-Kongresses beklagt hatte. ${ }^{28}$ Ein weiterer, nahezu typisch neokonservativer Argumentationsstrang be-

26 Condolezza Rice, »Promoting the National Interest « in: Foreign Affairs, 1/2000, S. 4562 (S. 61).

27 Stephen J. Solarz / Paul Wolfowitz, »Letter to the Editor « in: Foreign Affairs, 2/1999, in: <http://www.newamericancentury.org/iraq_pdf_01.pdf>. Zur Bedeutung der IrakFrage für die Neokonservativen seit Beginn der 1990er Jahre vgl. Stefan Fröhlich, »Hegemonialer Internationalismus« in: Frankfurter Allgemeine Zeitung, 10. 4. 2003.

28 Richard Perle, »Iraq: Saddam Unbound « in: Robert Kagan / William Kristol (Hg.), Present Dangers, aaO. (FN 20), S. 99-110: "If the next administration is to protect America's interests in the Gulf and help bring about the conditions for long-term stability in the region, it must formulate a comprehensive political and military strategy for bringing down Saddam and his regime. This can only be done by supporting the external opposition." (S. 107f.). 
züglich der amerikanischen Irak-Politik stellte die innere Verfasstheit des politischen Systems, d. h. die Menschenrechtsverletzungen Saddam Husseins und den totalitären Charakter des Baath-Regimes, in den Mittelpunkt. ${ }^{29}$ Schließlich trat nach dem 11. September noch ein zusätzliches Argument für einen Regimewechsel in Bagdad hinzu: Einige Neokonservative sehen nach wie vor trotz einer - vorsichtig formuliert - dürftigen Beweiskette Saddam Hussein unmittelbar in die Anschläge des 11. September verwickelt. ${ }^{30}$

Die Verbindung zwischen den beiden regionalen Ordnungen des Nahen und Mittleren Ostens sehen Neokonservative in einem Zusammenhang, der eine Variante oder vielmehr Umkehrung der »Domino-Theorie« darstellt: Wenn sich der Irak nach dem Sturz Saddam Husseins zu einem demokratischen, prosperierenden Staat entwickelte, würde dies eine Demokratisierungswelle im Nahen Osten auslösen, an deren Ende Regime dominierten, die auf Grund ihrer inneren Struktur keine aggressive Außenpolitik verfolgten. Israel könne daher sicher existieren, was wiederum der Regierung in Jerusalem diejenigen außen- und innenpolitischen Handlungsspielräume gäbe, einer endgültigen Regelung des israelisch-palästinensischen Konfliktes zuzustimmen. ${ }^{31}$

Zudem böte der Regimewechsel in Bagdad die Möglichkeit, auch die letzten amerikanischen Truppen aus Saudi-Arabien abzuziehen und damit einen der wichtigsten ideologischen Anknüpfungspunkte der al-Qaida zu beseitigen. Riad ist neben dem Irak im Mittleren Osten das bevorzugte Ziel neokonservativer Kritik, denn das politische System des langjährigen amerikanischen Verbündeten widerspricht, was die Gewährung der Menschenrechte und seinen Demokratisierungsgrad betrifft, fundamental neokonservativen Ordnungsvorstellungen. ${ }^{32}$ Eine Abwertung der bilateralen Beziehungen ist in der Amtszeit der Bush II-Administration bereits klar erkennbar, ein Regimewechsel in Riad wird nur deshalb nicht offen angestrebt, da unklar ist, welchen außenpolitischen Kurs ein vollständig demokratisiertes SaudiArabien einschlagen würde..$^{33}$ Vor diesem Hintergrund sind auch die gerade in Europa immer wieder ventilierten personellen Verbindungen der Bush-Administration zu saudischen Eliten den Neokonservativen ein Dorn im Auge.

Eher defensiv sind die Konturen neokonservativer Ordnungsvorstellungen bezüglich der amerikanischen Politik gegenüber Iran. Zwar sind der autoritäre Charakter dieses politischen Systems, seine Menschenrechtsverletzungen, seine aggres-

29 Vgl. dazu beispielhaft Lawrence F.Kaplan / William Kristol, The War over Iraq. Saddam's Tyranny and America's Mission, San Francisco 2003 sowie David Wurmser, Tyranny's Ally. America’s Failure to Defeat Saddam Hussein, Washington 1999.

30 Vgl. zu dieser Position Laurie Mylroie, The War Against America. Saddam Hussein and the World Trade Center Attacks: A Study of Revenge, New York 2001.

31 Zur Bedeutung von Elementen der Domino-Theorie in den außenpolitischen Vorstellungen der Bush-Administration vgl. die kritische Bestandsaufnahme von Jack Snyder, »Imperial Temptations « in: The National Interest, 71/2003, S. 29-40 (S. 34f.).

32 Vgl. Daniel Pipes, »The Scandal of U.S.-Saudi Relations« in: The National Interest, 70/ 2002-03, S. 66-78.

33 Vgl. Mark N. Katz, "What Do We Do If The Saudi Monarchy Falls? in: Comparative Strategy, 1/2003, S. 45-58. 
sive Ablehnung Israels und sein Nuklearwaffenprogramm die Grundlage für eine neokonservative Rhetorik, die auf einen Regimewechsel in Teheran zielt; in der Wahl der Mittel plädieren die entsprechenden Vordenker jedoch eher für Zurückhaltung. ${ }^{34}$ So lassen sich kaum neokonservative Plädoyers für ein militärisches Vorgehen der USA, vergleichbar dem gegen den Irak, finden. Stattdessen setzen die entsprechenden Vertreter auf die beispielhafte Wirkung eines demokratisierten Irak und eine daraus resultierende Stärkung der einheimischen iranischen Opposition, die letztlich zum Sturz des etablierten Systems führen werde..$^{35}$

\section{Fazit}

Bezüglich ihrer nah- und mittelöstlichen Ordnungsvorstellungen sind die Neokonservativen weder »Neo«, da die diesbezüglichen Prämissen und Handlungsanleitungen in allgemeiner Form seit mehr als zwanzig Jahren bekannt sind und in speziellerer Form, d. h. auf den Irak gemünzt, schon seit 1991 in Washington offen vorgetragen werden. Noch sind sie »konservativ«, im Gegenteil: Es ist Daniel Cohn-Bendit zuzustimmen, der Richard Perle während eines Streitgesprächs als einen »Revolutionär« bezeichnete: Die nahezu vollständige Neuordnung des Nahen und Mittleren Ostens entsprechend dem neokonservativen Wertekanon und mit dem entsprechenden Instrumentarium ist ein radikal-umstürzlerischer Entwurf, der die Konsequenzen des eigenen Handelns nicht immer ausreichend in Rechnung stellt, wie an den Problemen der amerikanischen Nachkriegspolitik im Irak abzulesen ist; der sicherlich schwerlich umsetzbar ist, da er die aus dem Sturz etablierter politischer Systeme resultierenden Entwicklungen zu wenig antizipiert und sich nur unzureichend darauf eingerichtet hat, der aber auf jeden Fall ambitioniert, kühn, ja - in einem analytischen Sinne des Wortes - visionär ist.

Vordergründig scheinen die Neokonservativen die amerikanische Gesellschaft sehr stark zu polarisieren. Der erste Blick täuscht jedoch. Denn bezüglich ihrer nahostpolitischen Konzeptionen mit ihrer nahezu vorbehaltlosen Unterstützung Israels befinden sich die Neokonservativen im Mainstream der amerikanischen Politik. ${ }^{36}$ Diese Parteinahme wird von weiten Teilen der öffentlichen Meinung, von einflussreichen gesellschaftlichen Gruppen, vor allem pro-israelischen Lobby-Organisationen der amerikanischen Juden, von religiösen Gruppen aus dem »Bible Belt« des amerikanischen Südens und Teilen der amerikanischen Gewerkschaften, vor allem aber von Mitgliedern des US-Kongresses geteilt. Dasselbe gilt seit dem 11.9.2001 auch für die neokonservativen Ordnungsvorstellungen für den Arabisch-Persischen

34 Vgl. z. B. Reuel Marc Gerecht, "Regime Change in Iran?« in: The Weekly Standard, 5. 8. 2002, S. 30-33 sowie ders., »Iran: Fundamentalism and Reform« in: Kagan, / Kristol, (Hg.), Present Dangers, aaO. (FN 20), S. 111-144.

35 Vgl. das Plädoyer Richard Perles für einen Regimewechsel in Teheran mit friedlichen Mitteln, in: »Konfrontation ist eben mein Job«, Interview mit Richard Perle, in: die tageszeitung, 20. 6. 2003.

36 Vgl. Zachary Selden, »Neoconservatives and the American Mainstream « in: Policy Review, April-May/ 2004, in: <http://www.policyreview.org/apr04/selden.html>. 
Golf, insbesondere für die amerikanische Irak-Politik. Diese Konzeptionen hatten bis dahin kein zentrales politisches Projekt der Bush-Administration dargestellt und wären innenpolitisch wahrscheinlich auch nicht durchsetzbar gewesen. Erst indem der transnational agierende Terrorismus und die Proliferation von Massenvernichtungswaffen sowohl unter politischen Eliten als auch in der politischen Öffentlichkeit zu den beiden wichtigsten sicherheitspolitischen Bedrohungen der USA wurden, die dann auch noch in der Person Saddam Husseins zusammenzulaufen schienen, verschob sich der politische Mainstream in Richtung neokonservativer Positionen und gab damit deren Vertretern die Handlungsspielräume zur Umsetzung längst vorhandener außenpolitischer Konzeptionen. ${ }^{37}$

Bezüglich der amerikanischen Nah- und Mittelostpolitik argumentieren die Neokonservativen weitgehend geschlossen. Zwar hält sie weltanschaulich lediglich eine lose Klammer zusammen, in ihren nahostpolitischen Konzeptionen sind die Neokonservative jedoch sehr homogen; das hier skizzierte Profil wird - soweit erkennbar - von allen den Neokonservativen zuzuordnenden Entscheidungsträgern geteilt. Man könnte der amerikanischen Nah- und Mittelostpolitik, vor allem der Irak-Frage, für eine lange Zeit sogar den Status einer spezifischen inhaltlichen Klammer, ja geradezu einen »unifying effect " für die Neokonservativen zuschreiben.

Diese bestimmen aber die Nahostpolitik der Bush-Administration nicht ausschließlich. Stattdessen tobt auch in der amerikanischen Nah- und Mittelostpolitik der aus anderen Politikfeldern bekannte Stellungskampf, der die Amtszeit Präsident Bushs bislang geprägt hat. ${ }^{38}$ Damit polarisieren die Neokonservativen den außenpolitischen Entscheidungsprozess und erschweren ihn damit.

Würden ausschließlich neokonservative Strategen die amerikanische Politik im arabisch-israelischen Konflikt bestimmen (hier kann der Einfluss der Neokonservativen prinzipiell als geringer eingeschätzt werden als bei der US-Politik am Arabisch-Persischen Golf), so wäre es unerklärlich, warum die USA z. B. ihre Politik zumindest deklaratorisch - in die Regelungsbemühungen der drei anderen Akteure des »Quartetts «, d. h. der EU, der UN und Russlands, einbetten; warum sie entgegen früheren Bekundungen Jassir Arafat in den Jahren 2003 und 2004 doch wieder faktisch als Akteur im Nahostfriedensprozess akzeptierten und die palästinensische Autonomiebehörde nach einer Unterbrechung von 18 Monaten im Jahr 2004 erneut finanziell unterstützen; und warum sie im Rahmen des Möglichen Kritik an der Politik der Regierung Scharon üben. Diese Schritte folgen keiner neokonservativen Perspektive, sondern sind nur mit dem Einfluss anderer Kräfte, allen voran der Beamten des State Department und des früheren Außenministers Colin Powell persönlich, zu erklären. Daneben stehen auch die Spitze des amerikanischen Militärs und weite Teile der Eliten der republikanischen Partei neokonservativen Ordnungs-

37 So Peter Rudolf, »Der 11. September, die Neuorientierung amerikanischer Außenpolitik und der Krieg gegen den Irak « in: Zeitschrift für Politik, 3/2003, S. 257-280.

38 Vgl. Elizabeth Drew, "The Neocons in Power in: The New York Review of Books, 10/ 2003, 12. 6. 2003, in: <www.nybooks.com/articles/16378>. 
vorstellungen für den Nahen und Mittleren Osten skeptisch bis ablehnend gegenüber. Auch in der Frage der Politik gegenüber Syrien und Iran dominieren gegenwärtig die Neokonservativen nicht die Agenda.

Zudem ist seit dem Sommer 2003 erkennbar, dass angesichts der fortdauernden militärischen Auseinandersetzungen im Irak, ausbleibender Demokratisierungsfortschritte im Nahen und Mittleren Osten und der anhaltenden Eskalation des israelisch-palästinensischen Konflikts die Neokonservativen an Legitimität in der amerikanischen Öffentlichkeit verlieren. Daher ist ihr Einfluss in der Öffentlichkeit spürbar im Rückgang begriffen und sie werden gerade von konservativer Seite scharf angegriffen. ${ }^{39}$ Aber auch innerhalb dieser Gruppe treten bezüglich der IrakPolitik inhaltliche Friktionen auf, die ihre Durchsetzungskraft schmälern. ${ }^{40}$ Die amerikanische Politik gegenüber dem Nahen und Mittleren Osten hat ihre o.a. einigende Wirkung auf die Neokonservativen mittlerweile verloren und sich im Gegenteil zu einem Spaltpilz innerhalb dieser Strömung entwickelt. ${ }^{41}$

Für eine vorsichtige Prognose bezüglich der Implementierung neokonservativer Ordnungsvorstellungen in der zweiten Amtszeit gilt es, das Verhältnis der personellen und strukturellen Determinanten zueinander zu bestimmen: Personell sind die Neokonservativen weder besonders gestärkt noch geschwächt: Ihre Vertreter haben keine höherrangigen Positionen erhalten als bisher; sowohl der Posten des Außenministers, der des Verteidigungsministers und der des Nationalen Sicherheitsberaters sind mit klassischen, am Realismus orientierten, Konservativen besetzt. Allenfalls ließe sich die Ablösung des neokonservativen Antipoden Colin Powell als eine Erweiterung ihrer Handlungsspielräume interpretieren. Bedeutsamer sind jedoch die strukturellen Faktoren, die umgekehrt auf die Barrieren für diese Strömung verweisen, ihre außenpolitischen Ordnungsvorstellungen umzusetzen: Das Window of Opportunity, das nach den Anschlägen vom 11. September 2001 der Administration außen- wie innenpolitisch solch große Handlungsspielräume eingeräumt hat, wie wenigen Präsidialadministrationen zuvor, hat sich wieder geschlossen und die verfassungsrechtliche Beschränkung der Exekutive durch die Legislative funktioniert wieder.

Zudem müssen sich außenpolitische Maßnahmen nicht zuletzt an ihrem Erfolg messen lassen und in diesen Fragen ist die neokonservative Bilanz alles andere als glänzend: Der arabisch-israelische Konflikt ist in den vergangenen vier Jahren durch

39 Vgl. stellvertretend Patrick Buchanan, Where the Right Went Wrong. How Neoconservatives subverted the Reagan Revolution and hijacked the Bush Presidency, New York 2004.

$40 \mathrm{Vgl}$. Thomas Kleine-Brockhoff, »Was ist und zu welchem Ende treibt Amerika? Die Wirklichkeit passt nicht zur Theorie - Amerikas Neokonservative streiten über die Lehren aus dem Irak-Krieg « in: Die Zeit, 30. 9. 2004.

41 Vgl. dazu G. John Ikenberry, »The End of the Neo-Conservative Moment « in: Survival, 1/2004, S. 7-22; Tim B. Müller, »Ihr Feind heißt Rumsfeld «, aaO. (FN 11); »Die Neokonservativen sind auf dem Rückzug«. Ein Gespräch mit Amitai Etzioni, in: Frankfurter Allgemeine Zeitung, 26. 9. 2003 und Ronald D.Asmus / Kenneth M. Pollack, »Werte statt Waffen. Bush und seine Neokonservativen sind im Irak gescheitert. Plädoyer für eine neue Strategie« in: Die Zeit, 4. 9. 2003. 
die USA seiner Regelung kein Schritt weiter zugeführt worden, im Gegenteil: Die zweite Intifada hat unter der israelischen und der palästinensischen Bevölkerung zahlreiche Opfer gefordert. Erste vorsichtige Annäherungen der Konfliktparteien seit dem November 2004 haben nichts mit der amerikanischen Nahostpolitik zu tun, sondern mit dem geplanten israelischen Truppenrückzug aus dem Gaza-Streifen - eine Maßnahme, die Neokonservative ablehnen müssen, da sie in ihrer Weltsicht terroristisches Verhalten belohnt - und mit dem Wechsel in der palästinensischen Führung nach dem Tod Jassir Arafats. Der Irak ist von der geplanten Demokratisierung und seiner Funktion als Transformationskern für die gesamte arabische Welt weit entfernt. In weiten Teilen des Landes ist staatliche Autorität nicht existent, ihre Legitimation wird gewaltsam bestritten und die amerikanischen Truppen werden auf Jahre zu einer Stationierung gezwungen sein. In Iran haben die Parlamentswahlen vom Februar 2004 und die Präsidentschaftswahlen 2005 unterstrichen, dass die Hoffnung eines Regimewechsels von innen die machtpolitischen Realitäten in dem Land verkennt. Auf Dauer werden die Vereinigten Staaten eine wahrscheinlich bald nuklear bewaffnete iranische Theokratie als Realität am Arabisch-Persischen Golf akzeptieren müssen. Saudi-Arabien ist von einer Demokratisierung und einer Akzeptanz westlicher Grundwerte weit entfernt und spielt bei der logistischen und finanziellen Unterstützung des transnationalen, islamistisch motivierten Terrorismus nach wie vor eine wichtige Rolle. Wo man im Nahen und Mittleren Osten auch hinschaut, hat die amerikanische Außenpolitik ihre eigenen Ziele nicht erreichen können, nicht weil sie in den vergangenen Jahren neokonservativen Empfehlungen nicht gefolgt wäre, sondern im Gegenteil, weil sie sich, zumindest teilweise, auf neokonservative Ordnungsvorstellungen eingelassen hat. Es gibt auch in der zweiten Bush-Administration Neokonservative, aber ob diesen vor dem skizzierten Hintergrund noch einmal entscheidender Einfluss auf die amerikanische Außenpolitik eingeräumt werden wird, muss bezweifelt werden.

\section{Zusammenfassung}

Der Aufsatz diskutiert am Beispiel des Nahen und Mittleren Ostens die außenpolitischen Ordnungsvorstellungen des amerikanischen Neokonservatismus. Dazu untersucht er zuerst, was sich substanziell hinter der politisierten Chiffre dieser intellektuellen Strömung verbirgt: Wo sind die Neokonservativen ideologisch zu verorten, auf welchem gesellschaftlichen Resonanzboden sind sie entstanden, und was ist das gemeinsame inhaltliche Band bezüglich der amerikanischen Außenpolitik? In einem zweiten Schritt werden die neokonservativen Positionen zur amerikanischen Weltpolitik analysiert und am Beispiel des Nahen und Mittleren Ostens bzw. der entsprechenden Politik der Administration von George W. Bush exemplifiziert. Abschließend diskutiert der Aufsatz das Verhältnis neokonservativer Ordnungsvorstellungen zu denen relevanter gesellschaftlicher Gruppen in den USA und gibt einen kurzen Ausblick, wie sich der neokonservative Einfluss in der amerikanischen Außenpolitik während der zweiten Amtszeit von Präsident Bush entwickeln könnte. 


\section{Summary}

The paper analyzes the foreign policy concepts of the neoconservative movement in the U.S. exemplifying them through the case study of the Middle East and the respective U.S. policy. Starting from the assumption that the term »neoconservative" is mostly used as a politicized term, the author discusses in a first step what is behind the label of the "neoconservative movement « in terms of political ideas: What is the historical and societal background of its rise since the 1970s and what is the common denominator of this heterogeneous group? In a second step the neoconservative foreign policy concepts will be analyzed as well as their influence on the foreign policy of the administration of George W. Bush. Finally, the paper discusses the relationship of the neoconservative movement to other societal actors in the US and gives a brief outlook on what to expect regarding the neoconservative influence in the second Bush administration. 